\title{
An Evidential Model of Multisensor Decision Fusion for Force Aggregation and Classification
}

\author{
Bin Yu, Joseph Giampapa, Sean Owens, Katia Sycara \\ School of Computer Science, Carnegie Mellon University \\ 5000 Forbes Avenue, Pittsburgh, PA 15213, USA \\ \{byu, garof, owens, katia\}@cs.cmu.edu
}

\begin{abstract}
-
This paper describes airborne sensor networks for target detection and identification in military applications. One challenge is how to process and aggregate data from many sensor sources to generate an accurate and timely picture of the battlefield. The majority of research in data fusion has focused primarily on level 1 fusion, e.g., using multisensor data to determine the position, velocity, attributes, and identity of individual targets. In this paper we present a novel approach to military force aggregation and classification using the mathematical theory of evidence and doctrinal templates. Our approach helps commanders understand operational pictures of the battlefield, e.g., enemy force levels and deployment, and make better decisions than adversaries in the battlefield. A simple application of our approach is illustrated in the simulated testbed OTBSAF and RETSINA system.
\end{abstract}

\section{INTRODUCTION}

Sensor networks are emerging as a new trend in information technology for monitoring and collecting information in both military and non-military applications. This paper describes airborne sensor networks for target detection and identification in military applications. In a military context, various airborne sensors, e.g., SAR (Synthetic Aperture Radar), EO (ElectroOptical radar), and GMTI (Ground Moving Target Indicator), are mounted on a number of platforms. These platforms such as an F-16 or UAV (unmanned aerial vehicle) are deployed in the battlefield for target detection, tracking, and classification. For example, a SAR or EO sensor can recognize the location and identity of a stationary target; a GMTI sensor can detect a moving target and track the movement of the target.

Over the past two decades, a large number of approaches to multisensor data fusion have been developed [1]. The majority of research in data fusion has focused primarily on sensor data alignment, association, and correlation in level 1 fusion, e.g., using multisensor data to determine the position, velocity, attributes, and identity of individual targets. For example, the identity declaration from each sensor usually provides multiple identity declaration and each of them is associated with a confidence factor. Given the identity declarations of individual targets from airborne sensors, data fusion techniques seek to process identity declarations from multiple sensors to achieve a joint declaration of identity [2].

One challenge in airborne sensor networks is how to process and aggregate data from many sources to generate an accurate and timely picture of the battlefield. Current attempts to bring more information of individual targets to commanders are doomed to failure due to cognitive overload. With enormous amounts of information available for command decisions, it is impossible for commanders to fully analyze raw information for corresponding situation assessment. A mechanism is required to allow commanders to easily model and assess the dynamic situations such as the behavior and intentions of enemy forces based on the flow and fusion of collected information from various sensors [3]. The understanding of the battlefield situation, including location, movement, and deployment of enemy forces, is essential for commanders to make better decisions than adversaries in the battlefield.

In this paper we present a novel approach to force aggregation and classification using the mathematical theory of evidence and doctrinal templates. A doctrinal template depicts the composition and deployment of various types of echelons. We assume the raw sensor data have been transformed into a consistent set of entities before multisensor identity fusion [2], [4], [5]. In particular, we will focus on the following three problems,

- How to interpret uncertain information from multiple sensors, so that we can fuse uncertain and inconsistent information for a target from different sensors, e.g., a T80 tank?

- How to recognize different types of echelons with the uncertain information for each target in a cluster of vehicles or for each subechelon in the echelon?

- What is the confidence level for a cluster of vehicles (echelons) if the cluster matches a doctrinal template, e.g., a T80 tank platoon?

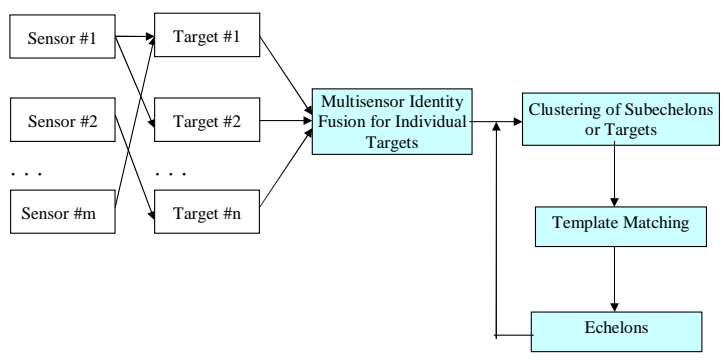

Fig. 1. The process of force aggregation and classification 


\begin{tabular}{|l|l|}
\hline USSR T80 & 0.4 \\
\hline \hline USSR T72M & 0.3 \\
\hline US M1 & 0.05 \\
\hline US M1A1 & 0.05 \\
\hline US M1A2 & 0.05 \\
\hline USSR 2S6 & 0.02 \\
\hline USSR ZSU23 4M & 0.03 \\
\hline US M977 & 0.001 \\
\hline US M35 & 0.001 \\
\hline US AVENGER & 0.001 \\
\hline US HMMWV & 0.001 \\
\hline USSR SA9 & 0.001 \\
\hline CLUTTER & 0.095 \\
\hline
\end{tabular}

(a)

\begin{tabular}{|l|l|}
\hline USSR T80 & 0.7 \\
\hline USSR T72M & 0.1 \\
\hline US M1 & 0.02 \\
\hline \hline US M1A1 & 0.02 \\
\hline US M1A2 & 0.02 \\
\hline USSR 2S6 & 0.01 \\
\hline \hline USSR ZSU23 4M & 0.02 \\
\hline US M977 & 0.001 \\
\hline US M35 & 0.001 \\
\hline US AVENGER & 0.001 \\
\hline US HMMWV & 0.001 \\
\hline USSR SA9 & 0.001 \\
\hline CLUTTER & 0.105 \\
\hline
\end{tabular}

(b)

\begin{tabular}{|l|l|}
\hline USSR T80 & 0.771 \\
\hline USSR T72M & 0.141 \\
\hline \hline US M1 & 0.016 \\
\hline US M1A1 & 0.016 \\
\hline US M1A2 & 0.016 \\
\hline \hline USSR 2S6 & 0.006 \\
\hline USSR ZSU23 4M & 0.013 \\
\hline US M977 & 0.0 \\
\hline US M35 & 0.0 \\
\hline US AVENGER & 0.0 \\
\hline US HMMWV & 0.0 \\
\hline USSR SA9 & 0.0 \\
\hline CLUTTER & 0.021 \\
\hline
\end{tabular}

Fig. 2. A low resolution sensor $S A R_{1}$ (a) and a high resolution sensor $S A R_{2}$ (b) return a list of candidate target types with different confidence levels for a ground target $T 80$ tank. The right table shows the fused confidence levels for each candidate target type.

Figure 1 describes the process of force aggregation and classification, including (1) multisensor identity fusion; (2) force aggregation (clustering); (3) force classification (template matching). Typically, the process of force aggregation is hierarchical. For example, a cluster of platoons can be clustered into a company that is further included at a battalion or a higher level force.

Commonly used algorithms for multisensor information fusion are Bayesian inference method and Dempster-Shafer theory of evidence. Dempster-Shafer theory produces identical results as Bayesian inference method for multisensor identity fusion when the hypotheses about individual target's identity declarations are singletons and mutually exclusive [6], [7], [8]. We choose Dempster-Shafer theory for the following two reasons,

- Most tactical sensors are incapable of assigning all confidences to each target type. Dempster-Shafer theory allows confidences to be assigned to sets of propositions rather than to just $N$ mutually exclusion propositions. For example, the confidence to clutter in the sensor data.

- Dempster-Shafer theory leads to the intuitive bottom-up process of force classification. The notion of conflict in Dempster-Shafer theory naturally captures the template matching process between a cluster of vehichles (or subechelons) and doctrinal templates.

The rest of this paper is organized as follows. Section 2 describes the approach for force aggregation and classification. Section 3 illustrates a simple application using the simulated testbed OTBSAF and RETSINA system. Section 4 summarizes the relevant literature. Section 5 concludes this paper and presents some directions for future research.

\section{Force Aggregation And Classification}

In the battlefield, various platforms are deployed to scan an area of terrain and attempts to recognize any stationary or moving targets within the bounds of that scanned area. The sensor output for each target is a list of candidate target types (e.g., M1 tank, T80 Tank, etc.) with different confidence levels. Our previous work considers the problem of force aggregation and classification based upon the sensor reports from two dissimilar sensors [9]. In this paper we extend the previous approach to the case of multiple sensors. We first describe how to combine the uncertain information from multiple sensor sources in the framework of Dempster-Shafer's theory, and then we present our approach to force aggregation and classification using Dempster-Shafer theory and doctrinal templates.

\section{A. Dempster-Shafer theory}

We now introduce the key concepts of the Dempster-Shafer theory. Let $V=\left\{v_{1}, v_{2}, \ldots, v_{n}\right\}$ be the set of possible vehicle types, where $v_{i}, 1 \leq i \leq n$, is the possible type of vehicles a SAR sensor can recognize (see Figure 2(a) and (b)). A frame of discernment $\Theta=\left\{v_{1}, v_{2}, \ldots, v_{n}\right\}$ is the set of hypotheses under consideration and $v_{i} \in V, 1 \leq i \leq n$.

Definition 1: Let $\Theta$ be a frame of discernment. A basic probability assignment (bpa) is a function $m$ : $2^{\Theta} \mapsto$ $[0,1]$ where $(1) m(\phi)=0$ ( $\phi$ is the empty set), and (2) $\sum_{\hat{A} \subseteq \Theta} m(\hat{A})=1$.

In this paper we consider a common frame of discernment for all SAR sensor outputs. The set of vehicle types $V$ is $\{T 80, T 72 M, M 1, M 1 A 1, M 1 A 2,2 S 6, Z S U 23, M 977, M 35$, $A V E N G E R, H M M W V, S A 9\}$. In our scenario, the basic probability assignment can be defined as follows: (1) for any target type $v_{i} \in V, m\left(\left\{v_{i}\right\}\right)=c\left(v_{i}\right)$, where $c\left(v_{i}\right)$ is the confidence level of $v_{i}$ in the table; (2) for any $\hat{A} \subseteq \Theta$ and $\hat{A} \notin V, m(\hat{A})=0$; (3) $m(\Theta)=c$ (clutter).

For a subset $\hat{A}$ of $\Theta$, the belief function $\operatorname{Bel}(\hat{A})$ is defined as the sum of the beliefs committed to the possibilities in $\hat{A}$. For individual members of $\Theta, \mathrm{Bel}$ and $m$ are equal, e.g., $\operatorname{Bel}(\{T 80\})=m(\{T 80\})$

A subset $\hat{A}$ of a frame $\Theta$ is called a focal element of a belief function Bel over $\Theta$ if $m(\hat{A})>0$. Given two belief functions over the same frame of discernment but based on distinct bodies of evidence, Dempster's rule of combination enables us to compute a new belief function based on the combined evidence. For every subset $\hat{A}$ of $\Theta$, Dempster's rule defines $m_{1} \oplus m_{2}(\hat{A})$ to be the sum of all products of the form $m_{1}(X) m_{2}(Y)$, where $X$ and $Y$ run over all subsets whose intersection is $\hat{A}$. 
Definition 2: (Dempster's rule of combination) Let $\mathrm{Bel}_{1}$ and $\mathrm{Bel}_{2}$ be belief functions over $\Theta$, with basic probability assignments $m_{1}$ and $m_{2}$, and focal elements $\hat{A}_{1}, \ldots, \hat{A_{k}}$, and $\hat{B}_{1}, \ldots, \hat{B}_{l}$, respectively. Suppose

$$
\sum_{i, j, \hat{A}_{i} \cap \hat{B}_{j}=\phi} m_{1}\left(\hat{A}_{i}\right) m_{2}\left(\hat{B}_{j}\right)<1
$$

Then the function $m: 2^{\Theta} \mapsto[0,1]$ that is defined by $m(\phi)=$ 0 , and

$$
m(\hat{A})=\frac{\sum_{i, j, \hat{A}_{i} \cap \hat{B}_{j}=\hat{A}} m_{1}\left(\hat{A}_{i}\right) m_{2}\left(\hat{B}_{j}\right)}{1-\sum_{i, j, \hat{A}_{i} \cap \hat{B}_{j}=\phi} m_{1}\left(\hat{A}_{i}\right) m_{2}\left(\hat{B}_{j}\right)}
$$

for all non-empty $\hat{A} \subset \Theta$ is a basic probability assignment [10].

Bel, the belief function given by $m$, is called the orthogonal sum of $\mathrm{Bel}_{1}$ and $\mathrm{Bel}_{2}$. It is written Bel $=\mathrm{Bel}_{1} \oplus \mathrm{Bel}_{2}$. Note that Dempster's rule of combination is associative and commutative. This means that the processes of combining evidence from multiple sensors are independent of the order in which the sensor outputs are combined.

For any $v_{i} \in V, m\left(\left\{v_{i}\right\}\right)$ can be simplified as

$$
\frac{m_{1}\left(\left\{v_{i}\right\}\right) m_{2}\left(\left\{v_{i}\right\}\right)+m_{1}\left(\left\{v_{i}\right\}\right) m_{2}(\Theta)+m_{2}\left(\left\{v_{i}\right\}\right) m_{1}(\Theta)}{1-\sum_{v_{j} \in V} m_{1}\left(\left\{v_{j}\right\}\right)\left(1-m_{2}\left(\left\{v_{j}\right\}\right)-m_{2}(\Theta)\right)}
$$

Similarly, we can compute $m(\Theta)$ as

$$
m(\Theta)=\frac{m_{1}(\Theta) m_{2}(\Theta)}{1-\sum_{v_{j} \in V} m_{1}\left(\left\{v_{j}\right\}\right)\left(1-m_{2}\left(\left\{v_{j}\right\}\right)-m_{2}(\Theta)\right)}
$$

Given a $T 80$ tank on the ground, Figure 2 describes a list of candidate target types with different confidence levels from a low resolution sensor $S_{1}$ and a high resolution sensor $S A R_{2}$. Figure 2 also gives the fused confidence levels for each candidate target type using Dempster's rule of combination. Sometimes, the information about targets from these airborne sensors is noisy. For example, a sensor may confuse a T80 tank with an $M 1 A 1$ tank and give a low confidence level for $T 80$ and give a high confidence level for $M 1 A 1$. Figure 3 illustrates two lists of candidate target types for a T80 tank using the same SAR sensor. The SAR sensor confuses the $T 80$ tank with an $M 1 A 1$ tank in list (b), where $m_{2}(\{T 80\})<$ $m_{2}(\{M 1 A 1\})$.

\begin{tabular}{|l|l|}
\hline USSR T80 & 0.4 \\
\hline USSR T72M & 0.3 \\
\hline US M1 & 0.05 \\
\hline US M1A1 & 0.05 \\
\hline US M1A2 & 0.05 \\
\hline USSR 2S6 & 0.02 \\
\hline USSR ZSU23 4M & 0.03 \\
\hline US M977 & 0.001 \\
\hline US M35 & 0.001 \\
\hline US AVENGER & 0.001 \\
\hline US HMMWV & 0.001 \\
\hline USSR SA9 & 0.001 \\
\hline CLUTTER & 0.095 \\
\hline
\end{tabular}

(a)

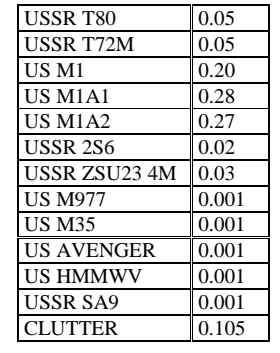

(b)
Fig. 3. The two lists of candidate target types for a $T 80$ tank using the same SAR sensor
For a given target $v$ on the ground, one interesting question is if we can get a higher confidence level for target $v$ after we fuse the two or more sensor reports from the same sensor or from sensors with similar resolutions. Figure 4 shows the fused confidence level for a $T 80$ tank when we fuse multiple sensor reports from the same sensor. The figure tells us that sometimes the confidence level for a $T 80$ tank could be very low from one single sensor report, e.g., $m(\{T 80\})=0.05$. We need to fuse multiple sensor reports to improve the accuracies of target classification. Also, the fused confidence level for the $T 80$ tank does not increase monotonically due to the noisy sensor data.

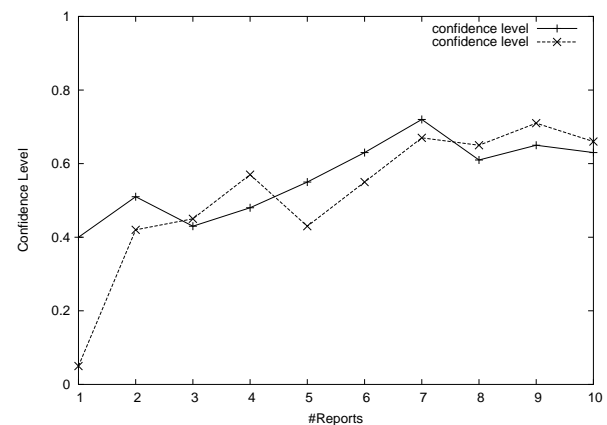

Fig. 4. The fused confidence level for a T80 tank using the same SAR sensor

\section{B. Clustering Algorithm for Force Aggregation}

The next step in force aggregation and classification is to identify candidate sets of vehicles to be considered as platoons or companies. We use a single-link agglomerative clustering algorithm to identify candidate sets of tanks to be considered as platoons or companies of tank. Different from other clustering algorithms like $k$-means algorithm, it does not require the number of clusters $k$ as an input, but needs a termination condition. The "single-link" approach, also called "nearest-neighbor" approach, starts with each tank or vehicle forming a separate group. It successively merges the objects or clusters according to the minimum distance between any two tanks $u_{a}$ and $u_{b}$, where $u_{a} \in S_{i}, u_{b} \in S_{j}, S_{i}$ and $S_{j}$ are two clusters. The clustering process is finished until all of the clusters are merged into one, or until the termination conditions meet [11].

Suppose we have observed $m$ tanks, $\left\{u_{1}, u_{2}, \ldots, u_{m}\right\}$ and we have estimated locations of the tanks. For tank $u_{i}$, let $x_{i}$ and $y_{i}$ denote the estimated $x$ and $y$ locations, respectively. The distance between any two tanks can be defined as

$$
d\left(u_{i}, u_{j}\right)=\sqrt{\left(x_{i}-x_{j}\right)^{2}+\left(y_{i}-y_{j}\right)^{2}}
$$

The distance between two sets of tanks $S_{i}, S_{j}$ is

$$
D\left(S_{i}, S_{j}\right)=\min _{u_{a} \in S_{i}, u_{b} \in S_{j}} d\left(u_{a}, u_{b}\right)
$$

Let $D\left(S_{i}, S_{j}\right)$ be the distance between cluster $S_{i}$ and $S_{j}$, and $N\left(S_{i}\right)$ is the nearest neighbor of cluster $S_{i}$. The singlelink clustering algorithm can be described as follows 
1) Initialize the clusters, where each cluster is a tank or vehicle in $\left\{u_{1}, u_{2}, \ldots, u_{m}\right\}$

2) For each pair of cluster $\left(S_{i}, S_{j}\right)$, compute $D\left(S_{i}, S_{j}\right)$

3) For each cluster $S_{i}$, compute $N\left(S_{i}\right)$

4) Repeat until we have the desired number of clusters or the termination conditions meet

a) Determine $S_{i}, S_{j}$ from existing clusters, such that $D\left(S_{i}, S_{j}\right)$ is minimized

b) Cluster $S_{i}$ and $S_{j}$

c) Update each $D\left(S_{i}, S_{j}\right)$ and $N\left(S_{i}\right)$ as necessary

5) End of the algorithm

The clustering of vehicles is based on the relative distance between any two vehicles (or clusters) and the number of vehicles in the template. For example, we know from the doctrinal templates that a platoon usually has 4 to 9 vehicles and these vehicles are deployed in a $100 m \times 100 m$ area. We will get clusters of tank platoons if we define the termination conditions as (1) the maximal $D\left(S_{i}, S_{j}\right)$ as $100 \mathrm{~m}$ and (2) maximal number of vehicles in a cluster as 9 . Sometimes the condition cannot guarantee we can identify clusters of vehicles at the low-level force, but we still can cluster them at highlevel. For example, if a company of T80 tank stays very close, we probably cannot recognize three $T 80$ platoons but we can recognize them as a $T 80$ company.

\section{Doctrinal Template Matching}

In this section we discuss how to recognize the type of an echelon using doctrinal templates. A doctrinal template depicts the composition and deployment of various types of subechelons or vehicles. For example, a T80 tank platoon template consists of four T80 tanks. A template may also have different kinds of vehicles. For example, an anti-tank platoon template consists of three tanks and six missile launchers. In general, a platoon template $T$ for an echelon can be represented as $T=\left\{v_{1}, v_{2}, \ldots, v_{p}\right\}$, where $v_{j} \in V(1 \leq j \leq p)$ is the type of a vehicle in template $T$. For each vehicle $v_{j}$, we assume the frame of discernment is $\left\{v_{j}\right\}$ and the corresponding basic probability assignment is $m\left(\left\{v_{j}\right\}\right)=1.0$.

The question is, given a cluster of vehicles $C L=\left\{u_{1}, \ldots\right.$, $\left.u_{q}\right\}$ and a list of doctrinal templates $\left\{T_{1}, T_{2}, \ldots, T_{l}\right\}$, how to determine the type of echelon. The basic idea here is to match the cluster of vehicles with each template. The matching process attempts to minimize the conflict between a template and cluster of vehicles. The template with the minimum conflict is the matched one for the cluster.

The matching process is simple if the template has same type of vehicles. As shown in Figure 5, each vehicle $u_{i}$ in the cluster CL is associated with a list of candidate target types and basic probability assignment $m_{i}$ for each target type and $\Theta$. Given a template $T_{k}=\left\{v_{1}, \ldots, v_{p}\right\}$, we can randomly match any vehicle $u_{i}$ with $v_{j}$ and then we sum up the conflict $C_{k}$ between $T_{k}$ and CL. The matching algorithm can be described as follows,

1) Initially, $C_{k}=0$.

2) $\forall u_{i} \in C L, \forall v_{j} \in T_{k}, C_{k}=C_{k}+1-m_{i}\left(\left\{v_{j}\right\}\right)-m_{i}(\Theta)$

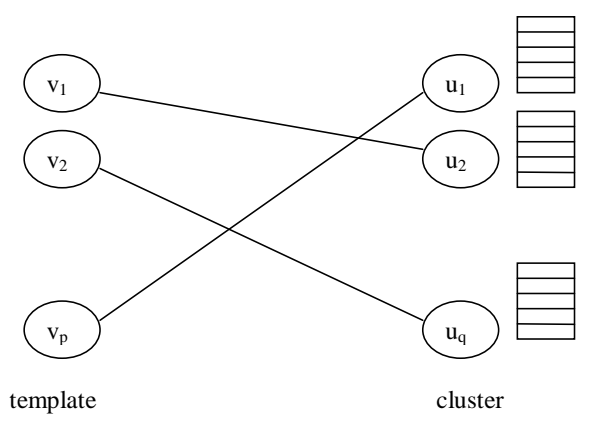

Fig. 5. The matching process between a template and a cluster of vehicles

3) $C L=C L-u_{i}$

4) $T_{k}=T_{k}-v_{j}$

5) if $C L=\phi$ or $T_{k}=\phi$, return $C_{k}=C_{k}+|C L|$

For templates with different types of vehicles, we have to consider the order of vehicles for matching, so that we can minimize the conflict between a template and the cluster. Obviously, the conflict is small if we match $u_{i}$ with the same type of vehicle $v_{j}$ in the template if $u_{i}$ has the maximal bpa for vehicle type $v_{j}$. For a target $u_{i}$, we denote $v_{j}=\Pi\left(u_{i}, 1\right)$ as the target type with the highest bpa, e.g., $m_{i}\left(\left\{v_{j}\right\}\right)>m_{i}\left(\left\{v_{k}\right\}\right)$ for any $v_{k}, v_{j} \in V$ and $v_{k} \neq v_{j}$. Similarly, we can define $\Pi\left(u_{i}, 2\right)$ as the target type with the second highest bpa.

During the first round of matching process, we choose $u_{i}$ with the maximal $m_{i}\left(\left\{\Pi\left(u_{i}, 1\right)\right\}\right)$ to match vehicle $v_{j}$ in a template. Formally, $\forall u_{k} \in C L, \Pi\left(u_{i}, 1\right)=\Pi\left(u_{k}, 1\right)$, and $u_{k} \neq u_{i}, m_{k}\left(\left\{\Pi\left(u_{k}, 1\right)\right\}\right) \leq m_{i}\left(\left\{\Pi\left(u_{i}, 1\right)\right\}\right)$, we call $u_{i}$ as the vehicle with the maximal probability assignments in CL, denoted as $u_{i}=\Gamma(C L, 1)$. Given a vehicle $u_{i} \in C L$ and $v_{j}$ is a vehicle in a template and $v_{j}=\Pi\left(u_{i}, 1\right)$, the conflict between $u_{i}$ and $v_{j}$ can be defined as $1-m_{i}\left(\left\{v_{j}\right\}\right)-m_{i}(\Theta)$.

Note that usually the matching process has to be iterated for several rounds. For example, sometimes the target type $\Pi\left(u_{i}, 1\right)\left(u_{i}=\Gamma(C L, 1)\right)$ does not match any vehicle in a template, e.g., $\Pi\left(u_{i}, 1\right) \notin T_{k}$. In this case we need to choose $u_{j}=\Gamma(C L, 2)$ to continue the matching. The process stops when either $C L$ or $T_{k}$ becomes empty.

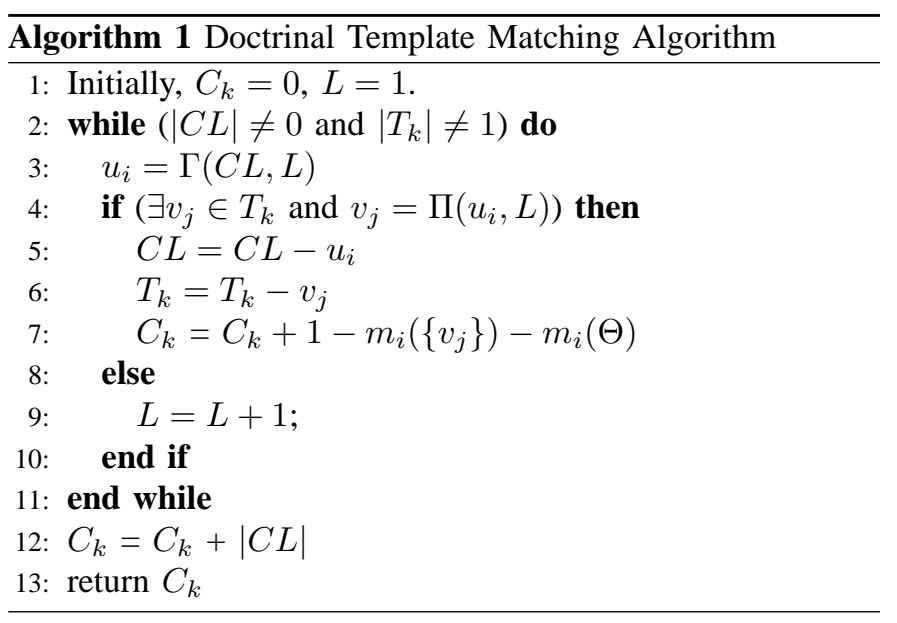


Algorithm 1 describes the process of matching a cluster of vehicles with a platoon template, where the conflict $C_{k}$ is initialized as 0 and the initial round $L$ is 1 . The algorithm can also be used to match high-level forces, e.g., a company, where each slot in the template or the cluster is a platoon.

The template with the minimum conflict with the cluster is the matched one. The confidence level of the matched template or unit type depends on the belief functions of each vehicles in the cluster. Here we use a belief function to represent the confidence level of the unit type and we give one way to compute the belief function of the matched unit type.

Definition 3: Given a cluster of vehicles $C L=\left\{u_{1}, u_{2}, \ldots\right.$, $\left.u_{q}\right\}$, assume $\left\{T_{1}, T_{2}, \ldots, T_{l}\right\}$ are templates for the force level, $T_{k}$ is the matched template with minimum conflicts with the cluster, $C_{i}$ is the conflict between $C L$ and any template $T_{i}$. We can get the corresponding basic probability assignment $m$ for $T_{i} \in\left\{T_{1}, T_{2}, \ldots, T_{l}\right\}$

$$
m\left(\left\{T_{i}\right\}\right)=\frac{\max \left(C_{1}, C_{2}, \ldots, C_{l}\right)-C_{i}}{\sum_{j=1}^{l}\left(\max \left(C_{1}, C_{2}, \ldots, C_{l}\right)-C_{j}\right)}
$$

The label of the $C L$ is $T_{k}$ if $m\left(\left\{\left(T_{k}\right\}\right)>m\left(\left\{T_{j}\right\}\right)\right.$ for any $T_{j} \in\left\{T_{1}, T_{2}, \ldots, T_{l}\right\}$ and $T_{k} \neq T_{j}$.

If we find three $T 80$ tanks stay together, we can infer that it is likely a $T 80$ tank platoon and its confidence level $m(\{$ T80Platoon $\})$ is high. Sometimes we may not find all three tanks; Instead, we only find two out of three or even one. The following figure illustrates the combined belief functions for three tank platoons with one, two, or three tanks. The figure tells us the confidence level is low if the sensors only find some of the total vehicles in templates. In practice, a T80 tank platoon with low confidence could be misclassified as an $M 1$ tank platoon at platoon level. However, the cluster of tanks can still be recognized as part of a $T 80$ tank company at company level using our approach. In other words, our approach is robust against the incomplete and ambiguous information from sensors and reduces effect of the ambiguity caused by missing targets on the higher level force classification.

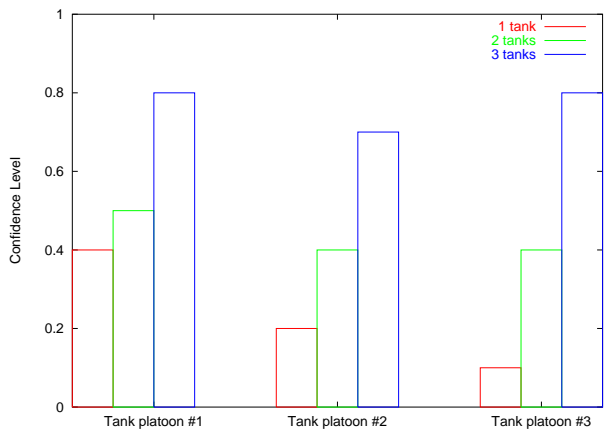

Fig. 6. The fused confidence level for a $T 80$ tank platoon with different number of tanks being found

\section{EXPERIMENTS}

In this section, we first introduce a modeling and simulation environment, OTBSAF (OneSAF Testbed Baseline) ${ }^{1}$ and its integration with the RETSINA system (Reusable Environment for Task Structured Intelligent Network Agents) [12]. And then we discuss some experimental results of force aggregation and classification in the simulated testbed.

\section{A. OTBSAF and RETSINA System}

OTBSAF models common military vehicles, aircraft, and sensors, and simulates uncertainty for entities' individual and doctrinal behaviors in the battlefield. We extend OTBSAF and integrate it with our RETSINA multiagent system. One of our contributions to OTBSAF is to add three simulated mounted sensors, SARSim, EOSim, and GMTISim, to the simulation environment.

The SARSim simulates an automatic target recognition (ATR) system that receives its input from a synthetic aperture radar (SAR) that is operating in spotlight-mode. In spotlightmode, a SAR scans an area of terrain, and the ATR will attempt to recognize any stationary object within the bounds of that scanned area. The output from the SARSim is a list of candidate target types (e.g., M1 tank, T80 Tank, etc.) with different confidence levels. While a real SAR/ATR system will report confidence levels for around three dozen entities, SARSim will report for a dozen entities. The GMTISim simulates a ground moving target indicator (GMTI) radar, which focuses a radar beam on one spot, and if it detects a moving target there with its ATR system, a motion tracker mechanism follows the movement of the target. While very similar in output and behavior to the SARSim, it is complementary, because it only recognizes entities that are moving, while the SARSim only recognizes entities that are stationary. The EOSim simulates an electro-optical sensor that detects targets at distances and in conditions in which they would be detectable in the ultraviolet, visible, and infrared light spectra.

\section{B. Experimental Results}

Let's consider the following scenario: an F-16 first locates targets in the battlefield using low resolution sensors, and then a UAV revisits some areas with groups of targets using high resolution sensors.

We consider a simple application of our approach for SARSims on the simulated testbed OTBSAF and RETSINA system, where there are three $T 80$ tank platoons, $P_{1}, P_{2}$, and $P_{3}$, on the ground, and each platoon consists of three $T 80$ tanks. An F-16 is tasked to scan the area first using a low resolution SARSim and then a UAV is tasked to scan the same area using a high resolution SARSim. Figure 7 and Figure 8 show a list of possible target identities with different confidence levels for each T80 tank on the ground. Note that the highest confidence identification in a series of lowconfidence is not necessarily the correct classification, e.g., target 1020, 1027, 1037, and 1044 in Figure 7. Also, tank

\footnotetext{
${ }^{1}$ http://www.onesaf.org/
} 


\begin{tabular}{|l|l|l|l|l|l|l|l|l|l|l|}
\hline & \multicolumn{3}{|c|}{ Cluster $\mathrm{P}_{1}$} & \multicolumn{3}{c|}{ Cluster $\mathrm{P}_{2}$} & \multicolumn{3}{c|}{ Cluster $\mathrm{P}_{3}$} & \\
\hline & 1014 & 1017 & 1020 & 1024 & 1027 & 1030 & 1034 & 1037 & 1040 & 1044 \\
\hline USSR T80 & 0.4 & 0.4 & 0.05 & 0.4 & 0.3 & 0.4 & 0.3 & 0.05 & - & 0.4 \\
\hline USSR T72M & 0.3 & 0.3 & 0.05 & 0.3 & 0.4 & 0.3 & 0.4 & 0.05 & - & 0.3 \\
\hline US M1 & 0.05 & 0.05 & 0.20 & 0.05 & 0.05 & 0.05 & 0.05 & 0.28 & - & 0.05 \\
\hline US M1A1 & 0.05 & 0.05 & 0.28 & 0.05 & 0.05 & 0.05 & 0.05 & 0.24 & - & 0.05 \\
\hline US M1A2 & 0.05 & 0.05 & 0.27 & 0.05 & 0.05 & 0.05 & 0.05 & 0.23 & - & 0.05 \\
\hline USSR 2S6 & 0.02 & 0.02 & 0.02 & 0.02 & 0.02 & 0.02 & 0.02 & 0.02 & - & 0.02 \\
\hline USSR ZSU23 & 0.03 & 0.03 & 0.03 & 0.03 & 0.03 & 0.03 & 0.03 & 0.03 & - & 0.03 \\
\hline US M977 & 0.001 & 0.001 & 0.001 & 0.001 & 0.001 & 0.001 & 0.001 & 0.001 & - & 0.001 \\
\hline US M35 & 0.001 & 0.001 & 0.001 & 0.001 & 0.001 & 0.001 & 0.001 & 0.001 & - & 0.001 \\
\hline US AVENGER & 0.001 & 0.001 & 0.001 & 0.001 & 0.001 & 0.001 & 0.001 & 0.001 & - & 0.001 \\
\hline US HMMWV & 0.001 & 0.001 & 0.001 & 0.001 & 0.001 & 0.001 & 0.001 & 0.001 & - & 0.001 \\
\hline USSR SA9 & 0.001 & 0.001 & 0.001 & 0.001 & 0.001 & 0.001 & 0.001 & 0.001 & - & 0.001 \\
\hline CLUTTER & 0.095 & 0.095 & 0.095 & 0.095 & 0.095 & 0.095 & 0.095 & 0.095 & - & 0.095 \\
\hline
\end{tabular}

Fig. 7. The confidence levels for tanks in a $T 80$ tank company from the outputs of the low resolution SAR sensor $S A R_{1}$ on an F-16. Tank 1044 is in the company but it does not belong to any platoon. "-" means the field is empty in the SARSim output.

\begin{tabular}{|l|c|c|c|c|c|c|c|c|c|c|}
\hline & \multicolumn{3}{|c|}{ Cluster $\mathrm{P}_{1}$} & \multicolumn{3}{c|}{ Cluster $\mathrm{P}_{2}$} & \multicolumn{3}{c|}{ Cluster $\mathrm{P}_{3}$} & \\
\hline & 1014 & 1017 & 1020 & 1024 & 1027 & 1030 & 1034 & 1037 & 1040 & 1044 \\
\hline USSR T80 & 0.7 & 0.7 & - & 0.7 & 0.7 & 0.7 & 0.7 & 0.7 & - & 0.7 \\
\hline USSR T72M & 0.1 & 0.1 & - & 0.1 & 0.1 & 0.1 & 0.1 & 0.1 & - & 0.1 \\
\hline US M1 & 0.02 & 0.02 & - & 0.02 & 0.02 & 0.02 & 0.02 & 0.02 & - & 0.02 \\
\hline US M1A1 & 0.02 & 0.02 & - & 0.02 & 0.02 & 0.02 & 0.02 & 0.02 & - & 0.02 \\
\hline US M1A2 & 0.02 & 0.02 & - & 0.02 & 0.02 & 0.02 & 0.02 & 0.02 & - & 0.02 \\
\hline USSR 2S6 & 0.01 & 0.01 & - & 0.01 & 0.01 & 0.01 & 0.01 & 0.01 & - & 0.01 \\
\hline USSR ZSU23 & 0.02 & 0.02 & - & 0.02 & 0.02 & 0.02 & 0.02 & 0.02 & - & 0.02 \\
\hline US M977 & 0.001 & 0.001 & - & 0.001 & 0.001 & 0.001 & 0.001 & 0.001 & - & 0.001 \\
\hline US M35 & 0.001 & 0.001 & - & 0.001 & 0.001 & 0.001 & 0.001 & 0.001 & - & 0.001 \\
\hline US AVENGER & 0.001 & 0.001 & - & 0.001 & 0.001 & 0.001 & 0.001 & 0.001 & - & 0.001 \\
\hline US HMMWV & 0.001 & 0.001 & - & 0.001 & 0.001 & 0.001 & 0.001 & 0.001 & - & 0.001 \\
\hline USSR SA9 & 0.001 & 0.001 & - & 0.001 & 0.001 & 0.001 & 0.001 & 0.001 & - & 0.001 \\
\hline CLUTTER & 0.105 & 0.105 & - & 0.105 & 0.105 & 0.105 & 0.105 & 0.105 & - & 0.105 \\
\hline
\end{tabular}

Fig. 8. The confidence levels for tanks in a $T 80$ tank company from the outputs of the high resolution SAR sensor $S A R_{2}$ on a UAV. Tank 1044 is in the company but it does not belong to any platoon. "-" means the field is empty in the SARSim output.

1020 is only found by sensor $S A R_{1}$ and tank 1040 is not found by either sensor.

Given SARSim outputs for a tank $u_{i}$, we first convert the outputs to belief functions and then combine the belief functions using Dempster's rule of combination. Next we discuss how to recognize the type of a given echelon solely based on the outputs of low resolution SARSim $S A R_{1}$, or based on the fused outputs of two SARsims $S A R_{1}$ and $S A R_{2}$ through Dempster-Shafer's theory. We assume tanks or vehicles have been clustered into platoons according to the distances between them.

1) Platoon Level Classification: In the platoon level, we choose 7 platoon templates from OTBSAF: US M1 platoon, US M1A1 platoon, US M1A2 platoon, USSR T72M platoon, USSR T80 platoon, USSR SA9 platoon, and USSR 2S6 platoon. Table I describes the number of each type of vehicles in different platoon templates.

Table II describes the conflicts of three clusters of tanks with platoon templates using Algorithm 1. Given the outputs of sensor $S A R_{1}$, clusters of tanks, $P_{1}$ and $P_{2}$, have minimal conflicts with $T 80$ platoon template, and can be classified as $T 80$ tank platoons. However, cluster $P_{3}$ has minimal conflict with T72M platoon template and is classified as a T72M tank

\begin{tabular}{|l|l|}
\hline Platoon templates & Vehicles \\
\hline \hline US M1 platoon & $4 \mathrm{M} 1 \mathrm{~s}$ \\
\hline US M1A1 platoon & $4 \mathrm{M} 1 \mathrm{~A} 1 \mathrm{~s}$ \\
\hline US M1A2 platoon & $4 \mathrm{M} 1 \mathrm{~A} 2 \mathrm{~s}$ \\
\hline USSR T72M platoon & $3 \mathrm{~T} 72 \mathrm{Ms}$ \\
\hline USSR T80 platoon & $3 \mathrm{~T} 80 \mathrm{~s}$ \\
\hline USSR SA9 platoon & 4 SA9s \\
\hline USSR 2S6 platoon & 2 2S6s \\
\hline
\end{tabular}

TABLE I

THE NUMBER OF EACH TYPE OF TANKS OR VEHICLES IN DIFFERENT PLATOON TEMPLATES

platoon, instead of a $T 80$ tank platoon. Note that our algorithm is tolerant to noisy sensor information. For example, although tank 1020 in $P_{1}$ is identified as an $M 1 A 1$ tank, we can still identify the cluster $P_{1}$ of two $T 80(1014,1017)$ and one $M 1 A 1$ (1020) as a $T 80$ tank platoon. Cluster $P_{3}$ is confused as a T72M tank platoon, since the sensor only finds two out of three tanks in the cluster and one of them is recognized as a T72M tank. In next section we will show our algorithm can still recognize the right type of echelon in the company level even with the outputs from the low resolution sensor. Also, 
if we match the three cluster of tanks with templates using the fused outputs from sensors $S A R_{1}$ and $S A R_{2}$, we find the results enhance the template matching, where the conflicts are minimized and all three clusters of tanks are classified as $T 80$ tank platoons.

\begin{tabular}{|l|l|l|l|l|l|l|}
\hline Templates & \multicolumn{2}{|c|}{$P_{1}$} & \multicolumn{2}{c|}{$P_{2}$} & \multicolumn{2}{c|}{$P_{3}$} \\
\hline \hline M1 pl. & 2.4 & $\mathbf{2 . 7}$ & 2.55 & $\mathbf{3}$ & 1.37 & $\mathbf{2}$ \\
\hline M1A1 pl & 2.32 & $\mathbf{2 . 6}$ & 2.55 & $\mathbf{3}$ & 1.41 & $\mathbf{2}$ \\
\hline M1A2 pl. & 2.33 & $\mathbf{2 . 6}$ & 2.55 & $\mathbf{3}$ & 1.42 & $\mathbf{2}$ \\
\hline T72M pl. & 2.05 & $\mathbf{2 . 5 7}$ & 1.7 & $\mathbf{2 . 5}$ & 1.25 & $\mathbf{1 . 7}$ \\
\hline T80 pl. & 1.85 & $\mathbf{1 . 3 1}$ & 1.6 & $\mathbf{0 . 7}$ & 1.35 & $\mathbf{0 . 8}$ \\
\hline SA9 pl. & 2.7 & $\mathbf{2 . 9}$ & 2.7 & $\mathbf{3}$ & 1.7 & $\mathbf{2}$ \\
\hline 2S6 pl. & 2.8 & $\mathbf{2 . 9}$ & 2.8 & $\mathbf{3}$ & 1.8 & $\mathbf{2}$ \\
\hline
\end{tabular}

TABLE II

THE CONFLICTS OF THREE CLUSTERS OF TANKS, $P_{1}, P_{2}, P_{3}$, WITH PLATOON TEMPLATES, WHERE THE CONFLICTS BASED ON FUSED OUTPUTS OF SENSORS $S A R_{1}$ AND $S A R_{2}$ ARE IN BOLD.

\begin{tabular}{|l|l|l|l|l|l|l|}
\hline Templates & \multicolumn{2}{|c|}{$P_{1}$} & \multicolumn{2}{c|}{$P_{2}$} & \multicolumn{2}{c|}{$P_{3}$} \\
\hline \hline M1 pl. & 0.13 & $\mathbf{0 . 0 7}$ & 0.08 & $\mathbf{0}$ & 0.19 & $\mathbf{0}$ \\
\hline M1A1 pl & 0.15 & $\mathbf{0 . 1 1}$ & 0.08 & $\mathbf{0}$ & 0.17 & $\mathbf{0}$ \\
\hline M1A2 pl. & 0.15 & $\mathbf{0 . 1 1}$ & 0.08 & $\mathbf{0}$ & 0.17 & $\mathbf{0}$ \\
\hline T72M pl. & 0.24 & $\mathbf{0 . 1 2}$ & 0.35 & $\mathbf{0 . 1 8}$ & 0.24 & $\mathbf{0 . 2}$ \\
\hline T80 pl. & 0.30 & $\mathbf{0 . 5 9}$ & 0.38 & $\mathbf{0 . 8 2}$ & 0.19 & $\mathbf{0 . 8}$ \\
\hline SA9 pl. & 0.03 & $\mathbf{0}$ & 0.03 & $\mathbf{0}$ & 0.04 & $\mathbf{0}$ \\
\hline 2S6 pl. & 0 & $\mathbf{0}$ & 0 & $\mathbf{0}$ & 0.0 & $\mathbf{0}$ \\
\hline
\end{tabular}

TABLE III

BASIC PROBABILITY ASSIGNMENTS FOR CLUSTERS OF TANKS, $P_{1}, P_{2}$, AND $P_{3}$, WHERE BASIC PROBABILITY ASSIGNMENTS BASED ON FUSED OUTPUTS OF SENSORS $S A R_{1}$ AND $S A R_{2}$ ARE IN BOLD.

The basic probability assignments for each cluster of tanks, $P_{1}$ and $P_{2}$, as a $T 80$ tank platoon can be computed according to Definition 3 and results are shown as in Table III, where frame of discernment for $P_{1}$ and $P_{2}$ is $\{$ T80_platoon, $\neg$ T80_platoon $\}$. Similarly, the basic probability assignments for $P_{3}$ are shown in the same table.

2) Company Level Classification: In this section we discuss the problem of recognizing the type of echelons in company level from platoons. We choose six templates in the company level: US M1 company, US M1A1 company, US M1A2 company, USSR T72M company, USSR T80 company, USSR $2 S 6$ battery (see Table IV). Some platoons are included in the template of a battalion level force directly, e..g, USSR SA9 platoon. Companies may have some extra vehicles besides the vehicles in the platoons. For example, a USSR T80 company has three T80 platoons and one extra T80 tank. In our experiments we do not consider the extra vehicles during template matching.

Table V describes the conflicts of the assumed T80 company with company templates. Obviously, the assumed $T 80$ company has the minimal conflicts with $T 80$ tank company template. The conflict with the $T 80$ company template changes to 0.79 when we use the fused outputs of sensors $S A R_{1}$

\begin{tabular}{|l|l|}
\hline Company templates & Platoons \\
\hline \hline US M1 company & 3 M1 platoons \\
\hline US M1A1 company & 3 M1A1 platoons \\
\hline US M1A2 company & 3 M1A2 platoons \\
\hline USSR T72M company & 3 T72M platoons \\
\hline USSR T80 company & 3 T80 platoons \\
\hline USSR 2S6 battery & 3 2S6 platoons \\
\hline
\end{tabular}

TABLE IV

THE NUMBER OF EACH TYPE OF PLATOONS IN DIFFERENT COMPANY TEMPLATES

\begin{tabular}{|l|l|l|}
\hline Templates & \multicolumn{2}{|l|}{ T80 company } \\
\hline \hline M1 company & 2.6 & $\mathbf{2 . 9 3}$ \\
\hline M1A1 company & 2.6 & $\mathbf{2 . 8 9}$ \\
\hline M1A2 company & 2.6 & $\mathbf{2 . 8 9}$ \\
\hline T72M company & 2.22 & $\mathbf{2 . 5}$ \\
\hline T80 company & 2.08 & $\mathbf{0 . 7 9}$ \\
\hline 2S6 battery & 3 & $\mathbf{3}$ \\
\hline
\end{tabular}

TABLE V

THE CONFLICTS OF THE ASSUMED T 80 COMPANY WITH COMPANY TEMPLATES, WHERE THE CONFLICTS BASED ON FUSED OUTPUTS OF SENSORS $S A R_{1}$ AND $S A R_{2}$ ARE IN BOLD.

and $S A R_{2}$. The basic probability assignments for the $T 80$ company are shown in Table VI

\begin{tabular}{|l|l|l|}
\hline Templates & \multicolumn{2}{|c|}{ T80 company } \\
\hline \hline M1 company & 0.14 & $\mathbf{0 . 0 2}$ \\
\hline M1A1 company & 0.14 & $\mathbf{0 . 0 4}$ \\
\hline M1A2 company & 0.14 & $\mathbf{0 . 0 4}$ \\
\hline T72M company & 0.27 & $\mathbf{0 . 1 6}$ \\
\hline T80 company & 0.31 & $\mathbf{0 . 7 4}$ \\
\hline 2S6 battery & 0 & $\mathbf{0}$ \\
\hline
\end{tabular}

TABLE VI

BASIC PROBABILITY ASSIGNMENTS FOR THE CLUSTER OF TANK PLATOONS, WHERE BASIC PROBABILITY ASSIGNMENTS BASED ON FUSED OUTPUTS OF SENSORS $S A R_{1}$ AND $S A R_{2}$ ARE IN BOLD.

\section{RELATED WORK}

The idea of using Dempster-Shafer theory for multisensor data fusion is not new. For example, Lowrance at al. apply Dempster-Shafer theory in reasoning about the locations and activities of multiple ships from intelligence reports [13]; Bogler studies whether the targets belong to the set of friendly or the set of not friendly aircraft in multisensor target identification systems using Dempster-Shafer theory [14]. Recently, Schubert extends those approaches to force clustering and classification [15], [16], where elements, e.g., intelligence reports, vehicles, and echelons, are clustered into subsets. Schubert uses the conflict of Dempster's rule as an indication of whether the elements belong together and focuses on how to handle intelligence reports with multiple nonspecific and uncertain hypotheses. Our work is related to Schubert's approach, but we do not consider sensor reports clustering for each target. 
Also, our approach is computationally efficient since we only consider pairwise conflicts between vehicles (or subechelon) in a cluster and elements in templates.

Bayesian inference techniques have been utilized for force aggregation [17]. Given the prior knowledge of each target and sensor in the battlefield, a Bayesian classifier has been developed for matching the observed echelon with different templates. For example, Bakert and Losiewicz partition the force into a mutually and exclusive set of units [18]. The units of the partition are used to create a set of unit templates in the hierarchy network of military force. In their approach, the posterior probability for each node is computing using Bayesian methods and is propagated through the hierarchy network as positive or negative evidence for the inclusion of each unit in the partition. In order to get better performance, the accumulated evidence in the network is learned through a set of randomly selected potential solutions using genetic algorithm. In this paper we use a bottom-up approach, instead of the top-down approach as described in [18], for force aggregation and classification. It would be interesting to study the possibility of combining these two approaches in force aggregation and classification.

Looney refined the fusion architecture for building a more accurate picture [19], [20]. Looney gave an alternative methods of fusing multisensor multitarget tracking data using a fuzzy clustering algorithm $-C$-means fuzzy clustering. Different from agglomerative clustering algorithm, fuzzy clustering assigns different degrees of membership to each entity. Thus, it allows each entity to belong to multiple clusters with various fuzzy membership values. Looney's approach makes the clustering independent of the ordering ( $k$-means algorithm relies on the order), but the number $K$ for a fuzzy $K$ cluster needs to be empirically estimated, where $K$ is usually unknown in the battlefield with asymmetric information. More recently, Looney and Liang developed a simple clustering algorithm for force aggregation, where each target is denoted as a feature vector [21]. The clustering algorithm is similar to ours, but they only consider the case of each target having a belief value between 0 and 1 for a single target type. In this paper, we describe a framework of multisensor data fusion for force aggregation, where the decision of a sensor is a list of candidate target types with different confidence levels.

\section{CONCLUSION}

An understanding of force level and deployment is essential for battlefield situation assessment and threat assessment. In this paper we present a novel approach to force aggregation and classification using Dempster-Shafer theory and doctrinal templates. In the future work we plan to evaluate the effectiveness of our approach at platoon and company level forces. We also plan to use the context of terrain and redundant sensor data to identify and reduce false positive targets in sensor reports [22].

\section{ACKNOWLEDGMENTS}

The authors would like to thank Robin Glinton, Charles Grindle, Dr. Michael Lewis and anonymous reviewers for their helpful comments. We would also like to thank our partners at Northrop-Grumman, led by Dr. Robert Mitchell, for their assistance in developing some of the external simulators. This research was supported by AFOSR under grants F49640-011-0542. and by AFRL/MNK grant No. F08630-03-1-0005.

\section{REFERENCES}

[1] D. L. Hall and J. Llinas, "An introduction to multisensor data fusion," in Proceedings of the IEEE, 1997, pp. 6-23.

[2] D. L. Hall, Ed., Mathematical Techniques in Multisensor Data Fusion. Artech House Inc., 1992.

[3] K. Sycara and M. Lewis, "From data to actionable knowledge and decision," in Proceedings of the Fifth International Conference on Information Fusion, 2002, pp. 577-584.

[4] E. Blasch and L. Hong, "Data association through fusion of target track and identification sets," in Proceedings of the Third International Conference on Information Fusion, 2000.

[5] L. A. Klein, Sensor and Data Fusion: Concepts and Applications. SPIE Optical Engineering Press, 1999.

[6] A. P. Dempster, "A generalization of bayesian inference," Journal of the Royal Statistical Society, 1968.

[7] J. J. Sudano, "Equivalence between belief theories and naive bayesian fusion for systems with independent evidential data: Part I, the theory," in Proceedings of the Sixth International Conference on Information Fusion, 2003, pp. 1239-1243.

[8] _ , "Equivalence between belief theories and naive bayesian fusion for systems with independent evidential data: Part II, the example," in Proceedings of the Sixth International Conference on Information Fusion, 2003, pp. 1357-1364.

[9] B. Yu, K. Sycara, J. Giampapa, and S. Owens, "Uncertain information fusion for force aggregation and classification in airborne sensor networks," in AAAI Workshop on Sensor Networks, 2004.

[10] G. Shafer, A Mathematical Theory of Evidence. Princeton, NJ: Princeton University Press, 1976.

[11] J. Han, M. Kamber, and A. K. Tung, "Spatial clustering methods in data mining: A survey," in Geographic Data Mining and Knowledge Discovery, H. J. Miller and J. Han, Eds. Taylor and Francis, 2001.

[12] K. Sycara, M. Paolucci, M. van Velsan, and J. Giampapa, "The RETSINA MAS infrastructure," Autonomous Agents and Multi-Agent Systems, vol. 7, no. 1, pp. 29-48, 2003.

[13] J. D. Lowrance, T. D. Garvey, and T. M. Strat, "A framework for evidential reasoning system," in Proceedings of the Fifth National Conference on Artificial Intelligence (AAAI), 1986, pp. 896-901.

[14] P. L. Bogler, "Shafer-dempster reasoning with applications to multisensor target identification systems," IEEE Transactions on System, Man, and Cybernetics, vol. 17, no. 6, pp. 968-977, 1987.

[15] J. Schubert, "Reliable force aggregation using a refined evidence specification from dempster-shafer clustering," in Proceedings of the Fourth International Conference on Information Fusion, 2001.

[16] — - "Evidential force aggregation," in Proceedings of the Sixth International Conference on Information Fusion, 2003, pp. 1223-1229.

[17] M. L. Hinman, "Some computational approaches for situation assessment and impact assessment," in Proceedings of the Fifth International Conference on Information Fusion, 2002, pp. 687-693.

[18] T. Bakert and P. B. Losiewicz, "Force aggregation via bayesian nodal analysis," in Proceedings of Information Technology Conference, 1998.

[19] C. G. Looney, "Exploring fusion architecture for a common operational picture," Information Fusion, vol. 2, no. 4, pp. 251-260, 2001.

[20] - "Interactive clustering and merging with a new fuzzy expected value," Pattern Recognition, vol. 35, no. 11, pp. 2413-2423, 2002.

[21] C. G. Looney and L. R. Liang, "Cognitive situation and threat assessments of ground battlespaces," Information Fusion, vol. 4, no. 4, pp. 297-308, 2003.

[22] R. Glinton, S. Owens, J. Giampapa, K. Sycara, C. Grindle, and M. Lewis, "Terrain based information fusion and inference," in Proceedings of the Seventh International Conference on Information Fusion, 2004, pp. 338-345. 